\title{
Exploring the powering source of the TeV X-ray binary LS 5039
}

\author{
Javier Moldón*, Marc Ribó, Josep M. Paredes \\ Departament d'Astronomia i Meteorologia and Institut de Ciències del Cosmos (ICC), \\ Universitat de Barcelona \\ Martí i Franquès, 1 \\ 08028 Barcelona, Spain \\ E-mail: jmoldon@am.ub.es, mribodam.ub.es, jmparedes@ub.edu

\section{Josep Martí} \\ Departamento de Física (EPS), Universidad de Jaén \\ E-mail: jmarti@ujaen.es
}

\section{Maria Massi}

Max Planck Institut für Radioastronomie

E-mail: mmassi@mpifr-bonn.mpg.de

LS 5039 is one of the four TeV emitting X-ray binaries detected up to now. The powering source of its multi-wavelength emission can be accretion in a microquasar scenario or wind interaction in a young non-accreting pulsar scenario. These two scenarios predict different morphologic and peak position changes along the orbital cycle of 3.9 days, which can be tested at milliarcsecond scales using VLBI techniques. Here we present a campaign of $5 \mathrm{GHz}$ VLBA observations conducted in June 2000 ( 2 runs five days apart). The results show a core component with a constant flux density, and a fast change in the morphology and the position angle of the elongated extended emission, but maintaining a stable flux density. These results are difficult to fit comfortably within a microquasar scenario, whereas they appear to be compatible with the predicted behavior for a non-accreting pulsar.

The 9th European VLBI Network Symposium on The role of VLBI in the Golden Age for Radio Astronomy and EVN Users Meeting

September 23-26, 2008

Bologna, Italy

\footnotetext{
* Speaker.
} 


\section{Introduction}

The very high energy emission of gamma-ray binaries (see [1, 2, 3, 丹) is basically interpreted as the result of inverse Compton upscattering of stellar UV photons by relativistic electrons. Two excluding scenarios have been proposed to explain the acceleration mechanism that powers the electrons. In the first one electrons are accelerated in the jets of a microquasar powered by accretion (see [5], [17], and [21]). In the second one they are accelerated in the shock between the relativistic wind of a non-accreting pulsar and the wind of the stellar companion (see [10], [8], and [22]).

LS 5039 is a gamma-ray binary situated at $2.5 \pm 0.5 \mathrm{kpc}$, composed of a bright young star and a compact object. The mass of the compact object depends on the inclination of the orbit, which can be between 11 and $75^{\circ}$, and is in the range $1.5-8 M_{\odot}$. The compact object can thus be a black hole or a neutron star [6].

\section{Testing the possible scenarios at mas scales and previous radio observations}

The expected behavior of the radio emission at mas scales is different in each scenario. In the microquasar scenario we have a central core with extended jet-like radio emission that displays a flux and distance asymmetry produced by projection effects and the Doppler boosting [13], [9]. The direction of the jets can display long-term precession. The jets can be bent or disrupted due to the interaction with the dense stellar wind of the bright companion [18]. In the non-accreting pulsar scenario the shocked material is contained in a 'bow' shaped nebula extending away from the stellar companion that follows an elliptical path during the orbital cycle. At mas scales, it is expected that the direction of the extended emission changes with the pulsar's orbital motion. The peak of the radio emission, located at a few AU behind the pulsar, should follow an elliptic orbit $\sim 10$ times bigger than the size of the orbit of the system [8].

The unresolved radio emission of LS 5039 is persistent, non-thermal, and variable, although no strong radio outburst or periodic variability have been detected so far [19, 7]. The source spectral index is $\alpha=-0.46 \pm 0.01$ [11]. No radio pulses have been detected at $1.4 \mathrm{GHz}$ [14]. However, free-free absorption with the stellar wind may prevent the detection of pulsations, and observations at higher frequencies are required. Observations at $5 \mathrm{GHz}$ were performed using the VLBA and the VLA in its phased array mode in 1999 [15]. The orbital phase of the system was in the range 0.12-0.15. The final synthesis map shows two-sided extended emission emerging from a central core with a flux density of $12.8 \mathrm{mJy}$, and aligned towards a direction with Position Angle (PA) $=125^{\circ}$, measured from north to east. The source extends over 6 mas on the plane of the sky. The morphology of the source was studied at larger scales with the EVN and MERLIN, also showing bipolar extended emission emanating from a central core (see Paredes et al. [16]).

\section{VLBA observations in June 2000}

We observed LS 5039 with the Very Long Baseline Array (VLBA) and the Very Large Array (VLA), of the National Radio Astronomy Observatory (NRAO), at $5 \mathrm{GHz}$ frequency on 2000 June 3 and 8 . The two 8-hour observing sessions, hereafter run A and run B, took place at orbital phases in the range $0.71-0.79$ and $0.43-0.51$, respectively. The observations were performed switching 
between the target source LS 5039 and the phase reference calibrator J1825-1718. The correlation position of J1825-1718 was shifted by $\Delta \alpha=+57.0$ mas and $\Delta \delta=+21.4$ mas from the accurate position provided by later observations from the joint NASA/USNO/NRAO geodetic/astrometric program. In the case of LS 5039, due to its proper motion, the source was found to be $\Delta \alpha=$ +7.6 mas and $\Delta \delta=-16.2$ mas away from the correlated position. See details in [20].

The post-correlation data reduction was performed using the Astronomical Image Processing System (AIPS). The VLA position had to be corrected by $0.43 \mathrm{~m}$, according to later geodetic measurements. The positions of J1825-1718 and LS 5039 were corrected using the task CLCOR. As recommended for phase-referencing experiments, we applied ionospheric and Earth Orientation Parameters corrections to the visibility data using CLCOR. The fringe fitting (FRING) of the residual delays and fringe rates was performed for all the sources. Fringes for 15 and $25 \%$ of the baselines were missing for the target source LS 5039 and for the astrometric check source J1837-1532, respectively. Typical data inspection and flagging were performed. An independent reduction of the VLA data was performed using standard procedures within AIPS.

\section{Results}

The VLA data correlated as an independent interferometer were compatible with a point-like source for the obtained synthesized beam of $5.5^{\prime \prime} \times 3.7^{\prime \prime}$ in PA $\sim-4^{\circ}$. We measured the flux density of the source every 30 minutes and obtained a mean of $29.4 \mathrm{mJy}$ with a standard deviation of $\sigma=1.1 \mathrm{mJy}$ during the 8 hours of run A, and $28.4 \mathrm{mJy}$ with $\sigma=0.7 \mathrm{mJy}$ for run B.

We show the final VLBA+phased VLA self-calibrated images in Fig. 1. The image obtained for run A displays a central core and bipolar and nearly symmetric extended emission with $\mathrm{PA} \simeq$ $116 \pm 2^{\circ}$, with the brightest component towards the south-east. The image is similar to the one obtained with the same array in 1999 May 8, corresponding to orbital phases $0.12-0.15$, which showed a slightly more asymmetric extended emission in PA $\sim 125^{\circ}$ (see [15]). In contrast, the image obtained for run B displays a core and a bipolar but clearly asymmetric structure with PA $\simeq 128 \pm 2^{\circ}$ and the brightest component towards the north-west. The total and peak flux densities fitted to the data are shown in Table 11. See also [20].

We splitted each run into 4-hour data sets, and self-calibrated each block. No significant morphological differences are measured between the two halves in any of the two runs. The peak position of the component SE1 with respect to Core 1 is stable in 4 hours within the errors $\left(\sigma_{\alpha}^{\mathrm{A}}=\right.$ 0.31 mas, $\left.\sigma_{\delta}^{\mathrm{A}}=0.62 \mathrm{mas}\right)$. For run B the distance between Core 2 and NW2 is also stable in 4 hours within the errors $\left(\sigma_{\alpha}^{\mathrm{B}}=0.55 \mathrm{mas}, \sigma_{\delta}^{\mathrm{B}}=0.25 \mathrm{mas}\right)$.

The measured position of LS 5039 for run A is $\alpha_{\mathrm{J} 2000.0}=18^{\mathrm{h}} 26^{\mathrm{m}} 15^{\mathrm{s}} .05653 \pm 0.15$ mas, and $\delta_{\mathrm{J} 2000.0}=-14^{\circ} 50^{\prime} 54^{\prime \prime} .2564 \pm 1.5$ mas. For checking purposes we measured the position of data blocks of 4 and 1-hour lengths. The peak position of LS 5039 appears to move $\Delta \alpha=+0.1 \pm$ 0.1 mas, $\Delta \delta=+2.8 \pm 0.2$ mas between the two 4-hour blocks (slightly more in the 1-hour blocks). The observed direction has a PA of $1.7 \pm 1.7^{\circ}$, which is the same as the line joining the positions of the source and J1825-1718. The expected error for differential astrometry is given by the separation $d$ in degrees from the phase-reference source and the offset $\Delta$ of its correlated position, according to $\Delta \times(d / 180) \times \pi$ (see [23]). Plugging our offset of 60.9 mas and a distance of 2.47 we obtain an error of 2.6 mas, very similar to the observed displacement of LS 5039. Similar results 

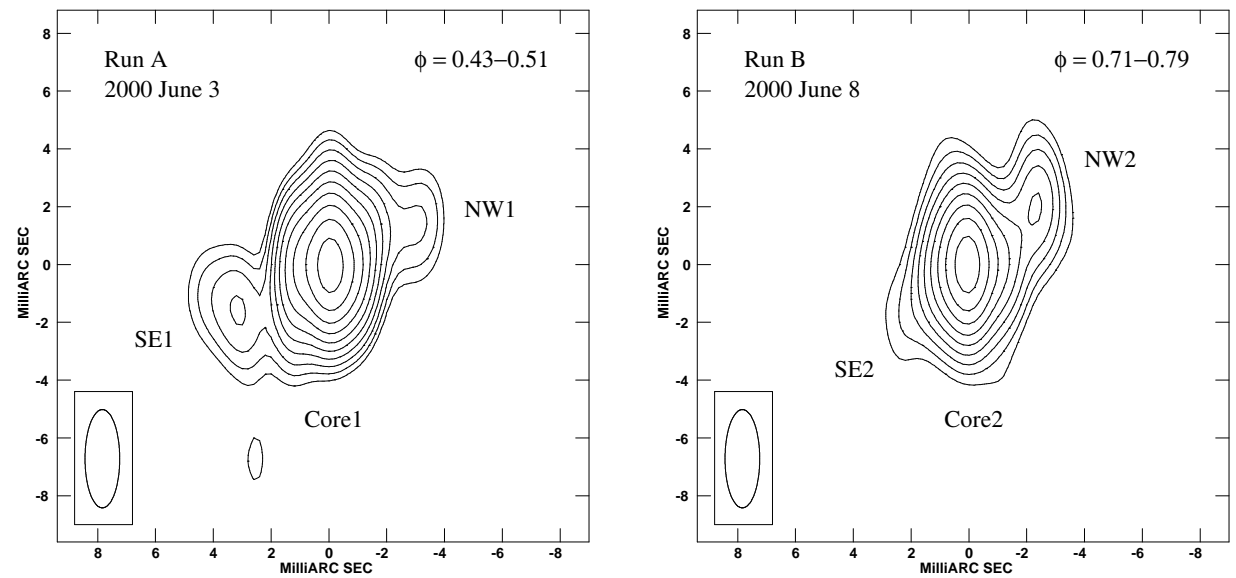

Figure 1: VLBA+phased VLA self-calibrated images of LS 5039 at $5 \mathrm{GHz}$ obtained on 2000 June 3 (left) and 8 (right). North is up and east is to the left. Axes units are in mas, and the $(0,0)$ position corresponds to the source peak in each image. The convolving beam, plotted in the lower left corner, has a size of $3.4 \times 1.2$ mas in PA of $0^{\circ}$. The first contour corresponds to 5 times the rms noise of the image $(0.08$ and $0.11 \mathrm{mJy} \mathrm{beam}^{-1}$ for run $\mathrm{A}$ and $\mathrm{B}$, respectively), while consecutive ones scale with $2^{1 / 2}$. The dates and orbital phases are quoted in the images. There is extended radio emission that appears nearly symmetric for run $\mathrm{A}$ and clearly asymmetric for run $\mathrm{B}$, with a small change of $\sim 12^{\circ}$ in its position angle.

Table 1: Parameters of the Gaussian components fitted to the data. Columns 3 and 4 list the peak and integrated flux densities of each component. Columns 5 to 8 list the polar and Cartesian coordinates of the components with respect to the peak position. The PA is positive from north to east.

\begin{tabular}{|c|c|c|c|c|c|c|c|}
\hline Run & Comp. & $\begin{array}{l}\text { Peak } S_{5} \mathrm{GHz} \\
{\left[\mathrm{mJy} \mathrm{beam}^{-1} \text { ] }\right.}\end{array}$ & $\begin{array}{l}S_{5 \mathrm{GHz}} \\
{[\mathrm{mJy}]}\end{array}$ & $\begin{array}{c}r \\
{[\mathrm{mas}]}\end{array}$ & $\begin{array}{l}\mathrm{PA} \\
{\left[{ }^{\circ}\right]}\end{array}$ & $\begin{array}{c}\Delta \alpha \\
{[\mathrm{mas}]}\end{array}$ & $\begin{array}{c}\Delta \delta \\
{[\mathrm{mas}]}\end{array}$ \\
\hline \multirow[t]{3}{*}{ A } & Core1 & $10.54 \pm 0.08$ & $20.0 \pm 0.2$ & - & - & - & - \\
\hline & SE1 & $1.11 \pm 0.08$ & $2.6 \pm 0.2$ & $3.67 \pm 0.08$ & $115.9 \pm 1.7$ & $3.30 \pm 0.07$ & $-1.60 \pm 0.12$ \\
\hline & NW1 & $0.88 \pm 0.08$ & $1.5 \pm 0.2$ & $3.29 \pm 0.09$ & $-63 \pm 2$ & $-2.92 \pm 0.08$ & $1.52 \pm 0.14$ \\
\hline \multirow[t]{3}{*}{ B } & Core2 & $10.45 \pm 0.11$ & $17.6 \pm 0.3$ & - & - & - & - \\
\hline & SE2 & $0.75 \pm 0.11$ & $1.8 \pm 0.4$ & $2.8 \pm 0.2$ & $129 \pm 5$ & $2.17 \pm 0.13$ & $-1.8 \pm 0.3$ \\
\hline & NW2 & $2.22 \pm 0.11$ & $3.9 \pm 0.3$ & $2.94 \pm 0.06$ & $-52.2 \pm 1.4$ & $-2.32 \pm 0.04$ & $1.80 \pm 0.09$ \\
\hline
\end{tabular}

are obtained when splitting the data from the astrometric check source J1837-1532. Therefore, these secular motions appear to be purely instrumental. The errors assigned to the coordinates of LS 5039 quoted above are half of the total secular motion measured in 1-hour blocks.

The phase-referenced image of run B reveals a double source with similar peak flux densities of $3.6 \pm 0.1 \mathrm{mJy}$ beam $^{-1}$, much lower than expected, and are separated $2.5 \pm 0.1$ mas in PA of $87 \pm 5^{\circ}$. Moreover, 4- and 1-hour blocks reveal a fading of the western component and a brightening of the eastern one along the run, as well as a similar secular motion as in run A. Tropospheric errors, which affect the phase-referenced image and cannot be accounted for, can easily split Core 2 into the observed double source. The precise position of the peak of LS 5039 cannot be measured in run $\mathrm{B}$. The relative astrometry between runs in these observations is therefore not useful. 


\section{Discussion}

The observations of LS 5039 reported here, obtained with the VLBA on two runs separated by 5 days, show a changing morphology at mas scales. In both runs there is a core component with a constant flux density within errors, and elongated emission with a PA that changes by $12 \pm 3^{\circ}$ between both runs. The brightest component in run A is towards south-east, and in run B towards north-west. The source is nearly symmetric in run A and asymmetric in run B (see Fig. 1). The relative astrometry obtained with current data is not considered in this discussion.

In the microquasar scenario, and assuming ballistic motions of adiabatically expanding plasma clouds without shocks [13], the morphology of run A can be interpreted as a double-sided jet emanating from a central core with the southeast component as the approaching one, whereas in run $\mathrm{B}$ the northwest component would be the approaching one. We can compute the projected bulk velocity of the jets from the distance and flux asymmetry. The results are compatible with a mildly relativistic jet with a projected bulk velocity of $0.1 \mathrm{c}$. However, the distances from Core 2 to the components NW2 and SE2 are very similar and do not imply any significant relativistic motion.

The lack of proper motions (see upper limits in Sect. 4) sets an upper limit to the projected bulk velocities of the components. For the measured flux asymmetries, the SE1 jet should be pointing at $\theta<48^{\circ}$, and the NW2 jet at $\theta<45^{\circ}$. In this context, a fast jet precession is needed to explain this behavior, and the PA of the jet should vary considerably, in contrast to the small range covered by all observed values at mas scale, between 115 and $140^{\circ}$ [15, 16]. See discussion in [20].

Alternatively, the morphology detected in run B could be the result of a discrete ejection where Core 2 is the approaching component and NW2 the receding one, while there is no radio emission at the origin of the ejection. However, large X-ray and radio flux density variations are expected during discrete ejections (see [ 8$]$ and references therein), while the peak and total radio flux densities of LS 5039 are strikingly constant (see also [19] and [7]) and there is no evidence of an X-ray flare in 11.5 years of RXTE/ASM data. Although not yet explored in detail, the morphology changes can be due to the interaction between the jets and a clumpy or dense stellar wind [18].

In the non-accreting pulsar scenario, the different morphologies we have detected at different orbital phases could be due to the change of the relative positions between the pulsar and the companion star along the orbit (see details in [8]). Observations at different orbital phases have always revealed a very similar PA for the extended emission, which would correspond to an inclination of the orbit as seen nearly edge on $\left(90^{\circ}\right)$. On the other hand, the absence of X-ray eclipses places an upper limit of $i \lesssim 75^{\circ}$ in this scenario. Therefore, these two restrictions imply an inclination angle that should be close to the upper limit of $75^{\circ}$.

In conclusion, a simple and shockless microquasar scenario cannot easily explain the observed changes in morphology. On the other hand, an interpretation within the young non-accreting pulsar scenario requires the inclination of the binary system to be very close to the upper limit imposed by the absence of X-ray eclipses. Precise phase-referenced VLBI observations covering a whole orbital cycle are necessary to trace possible periodic displacements of the peak position, expected in this last scenario, and to obtain morphological information along the orbit. These will ultimately reveal the nature of the powering source in this gamma-ray binary. 


\section{Acknowledgments}

The NRAO is a facility of the National Science Foundation operated under cooperative agreement by Associated Universities, Inc. J.M., M.R., J.M.P., and J.M. acknowledge support by DGI of the Spanish Ministerio de Educación y Ciencia (MEC) under grants AYA2007-68034-C03-01 and AYA2007-68034-C03-02 and FEDER funds. J.M. was supported by MEC under fellowship BES-2008-004564. M.R. acknowledges financial support from MEC and European Social Funds through a Ramón y Cajal fellowship. J.M. is also supported by Plan Andaluz de Investigación of Junta de Andalucía as research group FQM322. This work has benefited from research funding from the European Community's sixth Framework Programme under RadioNet R113CT 2003 5058187.

\section{References}

[1] Aharonian, F., Akhperjanian, A. G., Aye, K.-M., et al. 2005a, A\&A, 442, 1

[2] Aharonian, F., Akhperjanian, A. G., Aye, K.-M., et al. 2005b, Science, 309, 746

[3] Albert, J., Aliu, E., Anderhub, H., et al. 2006, Science, 312, 1771

[4] Albert, J., Aliu, E., Anderhub, H., et al. 2007, ApJ, 665, L51

[5] Bosch-Ramon, V., Romero, G. E., \& Paredes, J. M. 2006, A\&A, 447, 263

[6] Casares, J., Ribó, M., Ribas, I., et al. 2005, MNRAS, 364, 899

[7] Clark, J. S., Reig, P., Goodwin, S. P., et al. 2001, A\&A, 376, 476

[8] Dubus, G. 2006, A\&A, 456, 801

[9] Fender, R. P. 2006, Compact Stellar X-Ray Sources, ed. W. H. G. Lewin \& M. van der Klis (Cambridge University Press)

[10] Maraschi, L., \& Treves, A. 1981, MNRAS, 194, 1P

[11] Martí, J., Paredes, J. M., \& Ribó, M. 1998, A\&A, 338, L71

[12] Massi, M., Ribó, M., Paredes, J. M., et al. 2004, A\&A, 414, L1

[13] Mirabel, I. F., \& Rodríguez, L. F. 1999, ARA\&A, 37, 409

[14] Morris, D. J., Hobbs, G., Lyne, A. G., et al. 2002, MNRAS, 335, 275

[15] Paredes, J. M., Martí, J., Ribó, M., \& Massi, M. 2000, Science, 288, 2340

[16] Paredes, J. M., Ribó, M., Ros, E., Martí, J., \& Massi, M. 2002, A\&A, 393, L99

[17] Paredes, J. M., Bosch-Ramon, V., \& Romero, G. E. 2006, A\&A, 451, 259

[18] Perucho, M., \& Bosch-Ramon, V. 2008, A\&A, 482, 917

[19] Ribó, M., Reig, P., Martí, J., \& Paredes, J. M. 1999, A\&A, 347, 518

[20] Ribó, M., Paredes, J. M., Moldón, J., Martí, J., \& Massi, M. 2008, A\&A, 481, 17

[21] Romero, G. E., Torres, D. F., Kaufman Bernadó, M. M., \& Mirabel, I. F. 2003, A\&A, 410, L1

[22] Sierpowska-Bartosik, A., \& Torres, D. F. 2007, ApJ, 671, L145

[23] Walker, R. C. 1999, ASP Conf. Series, 180, 433 